\title{
Usefulness of Combining Galectin-3 and BIVA Assessments in Predicting Short- and Long-Term Events in Patients Admitted for Acute Heart Failure
}

\author{
Benedetta De Berardinis, ${ }^{1}$ Laura Magrini, ${ }^{1}$ Giorgio Zampini, ${ }^{1}$ \\ Benedetta Zancla, ${ }^{1}$ Gerardo Salerno, ${ }^{2}$ Patrizia Cardelli, ${ }^{2}$ Enrico Di Stasio, ${ }^{3}$ \\ Hanna K. Gaggin, ${ }^{4}$ Arianna Belcher, ${ }^{4}$ Blair A. Parry, ${ }^{5}$ John T. Nagurney, ${ }^{5}$ \\ James L. Januzzi Jr., ${ }^{4}$ and Salvatore Di Somma ${ }^{1}$ \\ ${ }^{1}$ Emergency Medicine, Department of Medical-Surgery Sciences and Translational Medicine, Sapienza University of Rome, \\ Sant'Andrea Hospital, Via di Grottarossa 1035/1039, 00189 Rome, Italy \\ ${ }^{2}$ Clinical and Molecular Medicine Department, Sapienza University of Rome, Sant'Andrea Hospital, 00189 Rome, Italy \\ ${ }^{3}$ Institute of Biochemistry and Clinical Biochemistry, Catholic University of the Sacred Heart, 00168 Rome, Italy \\ ${ }^{4}$ Division of Cardiology, Massachusetts General Hospital, Harvard Medical School, Boston, MA 02114, USA \\ ${ }^{5}$ Department of Emergency Medicine, Massachusetts General Hospital, Harvard Medical School, Boston, MA 02114, USA
}

Correspondence should be addressed to Salvatore Di Somma; salvatore.disomma@uniromal.it

Received 27 February 2014; Revised 15 May 2014; Accepted 16 May 2014; Published 30 June 2014

Academic Editor: Giulio Mengozzi

Copyright (C) 2014 Benedetta De Berardinis et al. This is an open access article distributed under the Creative Commons Attribution License, which permits unrestricted use, distribution, and reproduction in any medium, provided the original work is properly cited.

Introduction. Acute heart failure (AHF) is associated with a higher risk for the occurrence of rehospitalization and death. Galectin-3 (GAL3) is elevated in AHF patients and is an indicator in predicting short-term mortality. The total body water using bioimpedance vector analysis (BIVA) is able to identify mortality within AHF patients. The aim of this study was to evaluate the short- and longterm predictive value of GAL3, BIVA, and the combination of both in AHF patients in Emergency Department (ED). Methods. 205 ED patients with AHF were evaluated by testing for B type natriuretic peptide (BNP) and GAL3. The primary endpoint was death and rehospitalization at 30,60,90, and 180 days and 12 and 18 months. AHF patients were evaluated at the moment of ED arrival with clinical judgment and GAL3 and BIVA measurement. Results. GAL3 level was significantly higher in patients $>71$ years old, and with eGFR $<30 \mathrm{cc} / \mathrm{min}$. The area under the curve (AUC) of GAL3 + BIVA, GAL3 and BIVA for death and rehospitalization both when considered in total and when considered serially for the follow-up period showed that the combination has a better prognostic value. Kaplan-Meier survival curve for GAL3 values $>17.8 \mathrm{ng} / \mathrm{mL}$ shows significant survival difference. At multivariate Cox regression analysis GAL3 is an independent variable to predict death + rehospitalization with a value of $32.24 \mathrm{ng} / \mathrm{mL}$ at $30 \mathrm{days}$ $(P<0.005)$. Conclusion. In patients admitted for AHF an early assessment of GAL3 and BIVA seems to be useful in identifying patients at high risk for death and rehospitalization at short and long term. Combining the biomarker and the device could be of great utility since they monitor the severity of two pathophysiological different mechanisms: heart fibrosis and fluid overload.

\section{Introduction}

In patients with acute heart failure (AHF), the occurrence of rehospitalization and death is very common $[1,2]$. There is need for tools to immediately identify patients with AHF at high risk for short- and long-term mortality and readmission. Galectin-3 (GAL3) is a $\beta$-galactoside-binding lectin overexpressed by macrophages during phagocytosis that has been shown to be elevated in patients with AHF representing a prognostic biomarker for future adverse events such as death and rehospitalization $[3,4]$. The adverse outcome in patients with elevated circulating level of GAL3 has been linked with the presence of enhanced amount of the fibrosis of the heart [5]. Between patients that need to be hospitalized for 
AHF the occurrence of body congestion is a common finding [6].

Recently, the assessment of total body water using bioimpedance vector analysis (BIVA) has been suggested to be useful for the differential diagnosis of dyspnea, identifying patients with fluid overload [7]. Moreover, within subjects referring to the Emergency Department (ED) for AHF, BIVA has been demonstrated to be able to identify people at high risk for short-term mortality $[8,9]$. In consideration of the two different aspects of GAL3 and of BIVA (the first is a fibrosis marker, and the second is a dynamic marker of congestion), we decided to evaluate in this study the degree of congestion correlated to that of fibrosis in AHF patients alone or together for the prediction of events. So far no data are available on potential usefulness of combining GAL3, as a biomarker of heart fibrosis, and BIVA, as a device for detecting fluid overload in patients with AHF in order to identify subject at high risk for future adverse outcome. The aim of this study was to evaluate the short- and long-term predictive value of GAL3, BIVA, and the combination of both in patients with AHF at the moment of their hospital admission.

\section{Materials and Methods}

2.1. Study Design. In a prospective, blinded international study, patients presenting to ED with AHF were evaluated by testing for B type natriuretic peptide (BNP) and GAL3. The primary endpoint was death and rehospitalization at 30, 60, 90, and 180 days and 12 and 18 months.

We enrolled 205 subjects from March 2012 to September 2013 at two tertiary care academic medical centers members of the Global Research on Acute Conditions Team (GREAT): Sant'Andrea Hospital (Rome, Italy) and the Massachusetts General Hospital (Boston, MA). All study procedures were approved by local institutional review boards. AHF patients were evaluated at the moment of ED arrival with clinical judgement and blood routine laboratory tests plus GAL3 and BIVA. Inclusion criteria included moderate or severely symptomatic AHF (classified on the basis of current guidelines [10]) requiring intensification of diuretic therapy [10]. Exclusion criteria included renal failure requiring current renal replacement therapy, $\geq 8$ hours from the first dose of intravenous diuretic, and unwillingness or inability to participate in study procedures. At the moment of ED arrival, baseline demographics, vital signs, and results of physical examination were evaluated and recorded after informed consent was signed. The protocol was designed following the criteria of the Declaration of Helsinki and was approved by the ethical committee of each participating hospital. Peripheral venous blood was withdrawn and processed as noted below.

2.2. Blood Analysis. Peripheral venous blood was withdrawn by each patient and put into tubes containing ethylenediaminetetraacetic acid or no anticoagulant and spun for 15 minutes; samples were immediately aliquoted to freezer tubes and frozen at $-80^{\circ}$ for biomarkers measurement following the completion of the trial. Samples were thawed for the first time for measurement of biomarkers. Biomarkers of myocardial stretch BNP (Alere Triage BNP, San Diego, CA), biomarkers of risk stratification of patients GAL3 (VIDAS, Biomerieux, Marcy l'Étoile, France), and biomarkers of renal function included blood urea nitrogen (BUN), serum creatinine, and estimated glomerular filtration rate (eGFR, estimated using the simplified modification of diet in renal disease equation [11]). Clinicians were blinded to GAL3 and BIVA results.

2.3. Galectin-3. GAL3 (VIDAS, Biomerieux, Marcy l'Étoile, France) is a quantitative, one-step sandwich assay with fluorescence detection, designed for use with the VIDAS automated immunoassay system. Briefly the system measures GAL3 in human serum or plasma $(200 \mu \mathrm{L})$ using the ELFA (enzyme-linked fluorescent assay) technique in 20 minutes. All stages of the assay are performed automatically by the instrument, calculating the concentration of GAL3 relative to a stored calibration curve and enabling patients to be assigned as low, intermediate, or high risk of GAL3 mediated HF. Correct assay performance and validation of results are ensured by analysis of the control sample included in the kit. Decisional cut-offs are represented by $\leq 17.8 \mathrm{ng} / \mathrm{mL}$ "low risk," $17.8-25.9 \mathrm{ng} / \mathrm{mL}$ "intermediate risk," and $\geq 25.9 \mathrm{ng} / \mathrm{mL}$ "high risk" [12]; the three risk categories have been previously defined for the BGM Galectin-3 microplate assay [13].

2.4. BIVA Assessment. We used standard tetrapolar bioelectrical impedance electrodes at a frequency of $50 \mathrm{kHz}$ (Akern Srl, Pontassieve, Florence, Italy). The BIVA measurement assessed at patients' ED arrival was performed at bedside, with the patient supine, without metal contacts, and with inferior limbs at $45^{\circ}$ and superior limbs abducted at $30^{\circ}$ to avoid skin contacts. Four skin electrodes were applied (two on the wrist and two on the ipsilateral ankle) maintaining a minimal interelectrode distance of $5 \mathrm{~cm}$. The machine used an alternating current flux of $300 \mu \mathrm{A}$ and an operating frequency of $50 \mathrm{kHz}$. The results were visualized in two ways: as a vector or as a BIVA-derived hydration percentage. The first method includes a direct impedance plot which measures resistance $(\mathrm{Rz})$ and reactance $(\mathrm{Xc})$ as a bivariate vector in a nomogram. Reference values adjusted for age, $\mathrm{BMI}$, and gender are plotted as tolerance ellipses in the same coordinate system. Three tolerance ellipses are distinguished, corresponding to the 50th, 75th, and 95th vector percentiles of the healthy reference population. The major axis of this ellipse indexes hydration status and the minor axis reflects tissue mass. The second method involves a scale called a hydrograph (or hydrogram), which expresses the state of hydration as a percentage (HI). This value is calculated by an independently determined equation that uses the two components of BIVA, $\mathrm{Rz}$ and Xc. The normal value is $73.3 \%$ with tolerance between $72.7 \%$ and $74.3 \%$, corresponding to the 50th percentile. On arrival at the ED, $\mathrm{Rz}$ and $\mathrm{Xc}$ were recorded, normalized by the subject's height, and graphically expressed on the Rz-Xc plane; furthermore, HI was also assessed [8, 14-17]. Clinicians were blinded to the results of BIVA. 


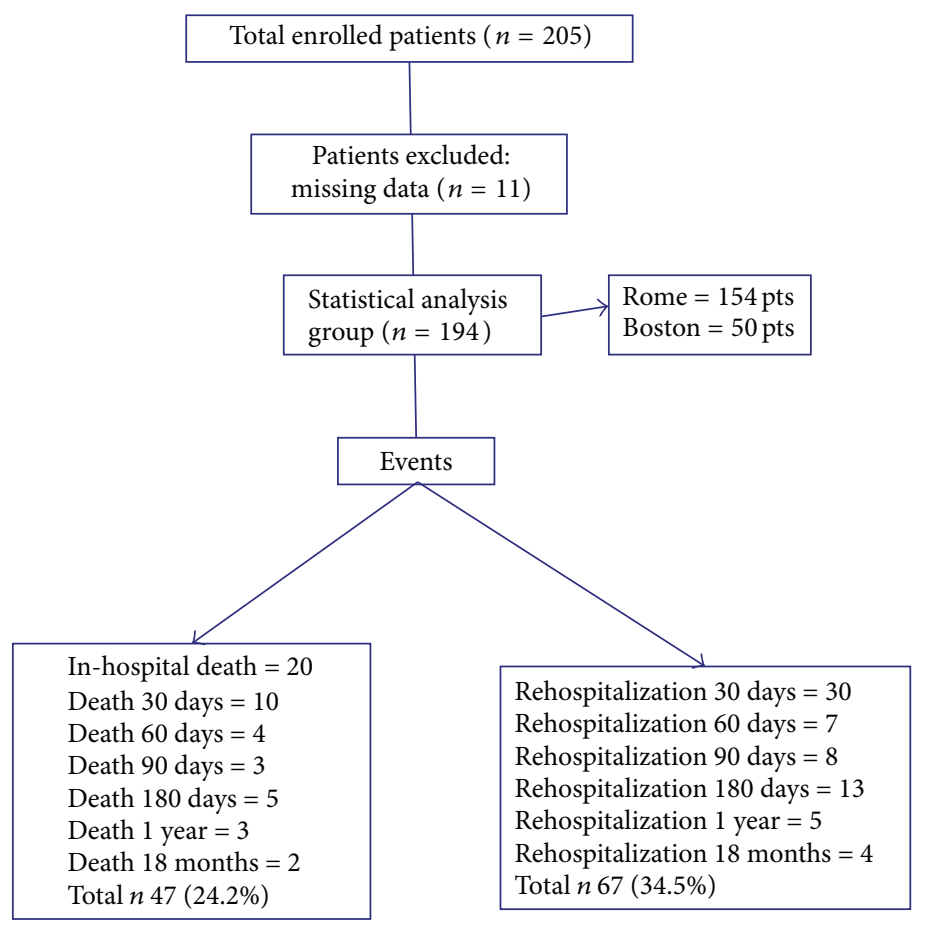

FIGURE 1: Flowchart of the study.

2.5. Follow-Up. In-hospital, 30-, 60-, 90-, and 180-day and 12and 18-month follow-up events (deaths or rehospitalization) were recorded.

2.6. Statistical Analysis. Continuous variables were summarized as mean \pm standard deviation (SD) if normally distributed and as median and interquartile range [IQR] if not normally distributed. Discrete variables are shown as percentage. Baseline variables of study participants for events (death + rehospitalization) and death were compared using Student's $t$-test for continuous variables or $X_{2}$ test for discrete variables if data were normally distributed; the Mann-Whitney $U$ test was used for continuous variables and Fisher's exact test for discrete variables in the states of nonnormality. To determine the prognostic value of GAL3 and phase angle, the receiver operating characteristic (ROC) tests compared the results of GAL3 and phase angle and combination of both for predicting rehospitalization or death, expressed as area under the curve (AUC); the $P$ value was obtained. Univariable comparisons between baseline characteristics were used to identify candidate variables for entry to a multivariable logistic regression model in order to select the variables most predictive of patients' outcomes; only those with a $P$ value $<0.05$ were retained for multivariable modeling. Net reclassification index (NRI) analysis was used to improve the accuracy of the risk-prediction model for in-hospital mortality. All statistical analyses were performed using Medcalc version 12.1.4 (Medcalc Software, Mariakerke, Belgium) software. All $P$ values are two-sided with a value of $<0.05$ considered significant.

\section{Results}

Figure 1 showed the flowchart of the study, 11 patients were excluded for missing data, total deaths in hospital and during 18-month follow-up were 47 (24.2\%), and total rehospitalization during all follow-up period was 67 (34.5\%). Patients' characteristics are showed in Table 1 . At the moment of ED arrival compared to survivors there were no statistically significant differences for demographic data in patients with death or total adverse events (rehospitalization and death) observed in all periods of the study. Beta blockers use was higher in patients who survived. On the contrary, in patients who died, there was a significant increase of serum creatinine ( $\mathrm{sCr}$ ), blood urea nitrogen (BUN), and white blood cells (WBC). When considering the combination of death and rehospitalization during follow-up period, there was significant increase of age, hypertension, diabetes mellitus, use of ACE inhibitors (ACEi), sCr, and $\mathrm{BUN}$ values in patients who develop events. Table 2 shows that, compared to survivors, GAL3 level at admission was significantly higher in both groups that died or that developed death + rehospitalization during follow-up. On the contrary, BNP value was not different within groups of patients who survived, died, or were rehospitalized. As for BIVA data, there was a significant increase of Xc in patients who died during followup compared to survivors (Table 2). Figure 2 shows the value of GAL3 subdivided in quartiles on the basis of age (a) and of eGFR (b). Figure 2(a) demonstrates that GAL3 level was significantly higher in patients within quartiles of age $>71$ years and in patients within quartiles of eGFR $<30 \mathrm{~mL} / \mathrm{min} / 1.73 \mathrm{~m}^{2}$ (Figure 2(b)). Table 3 shows the ROC curve analysis of GAL3, 


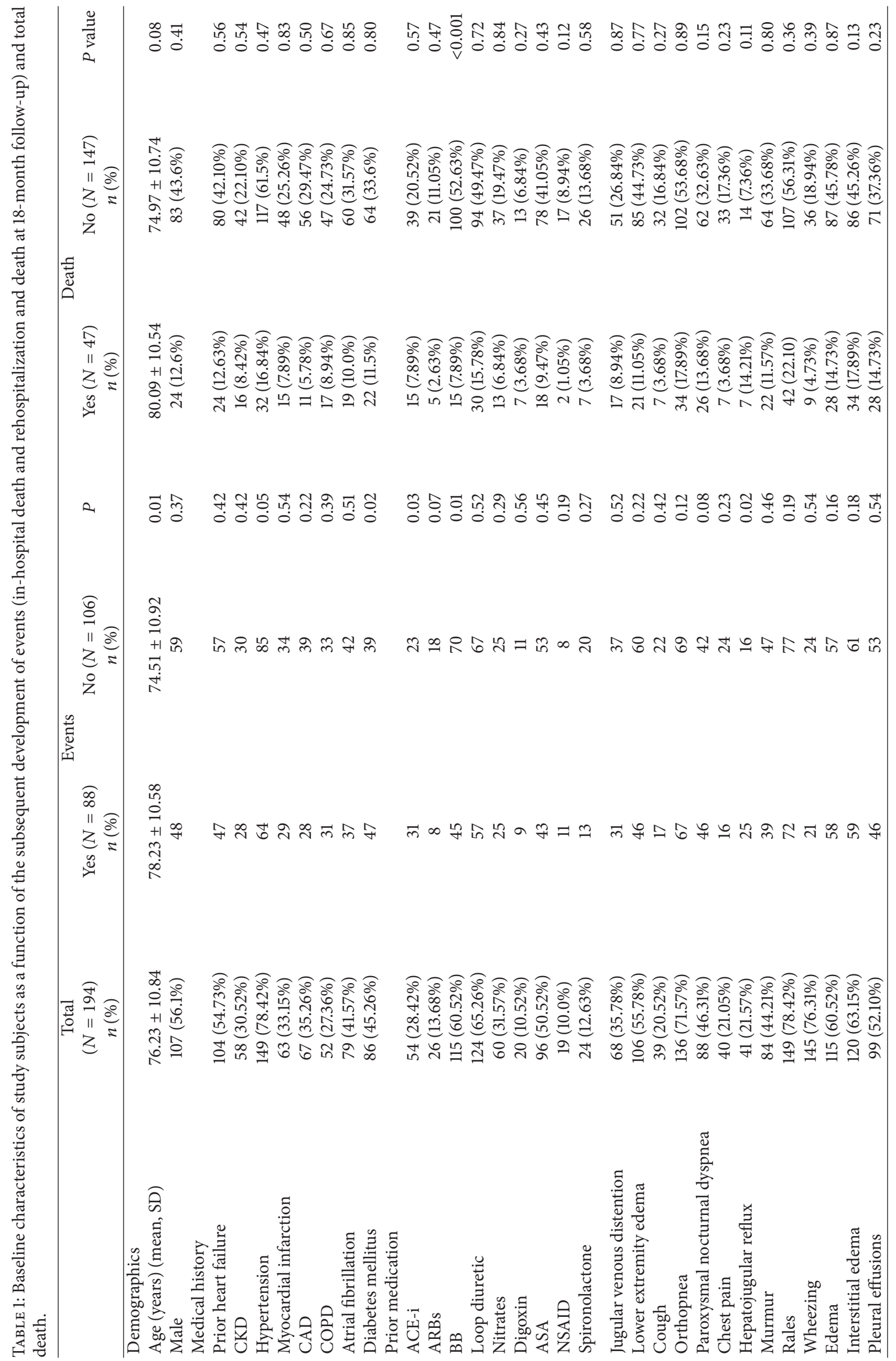




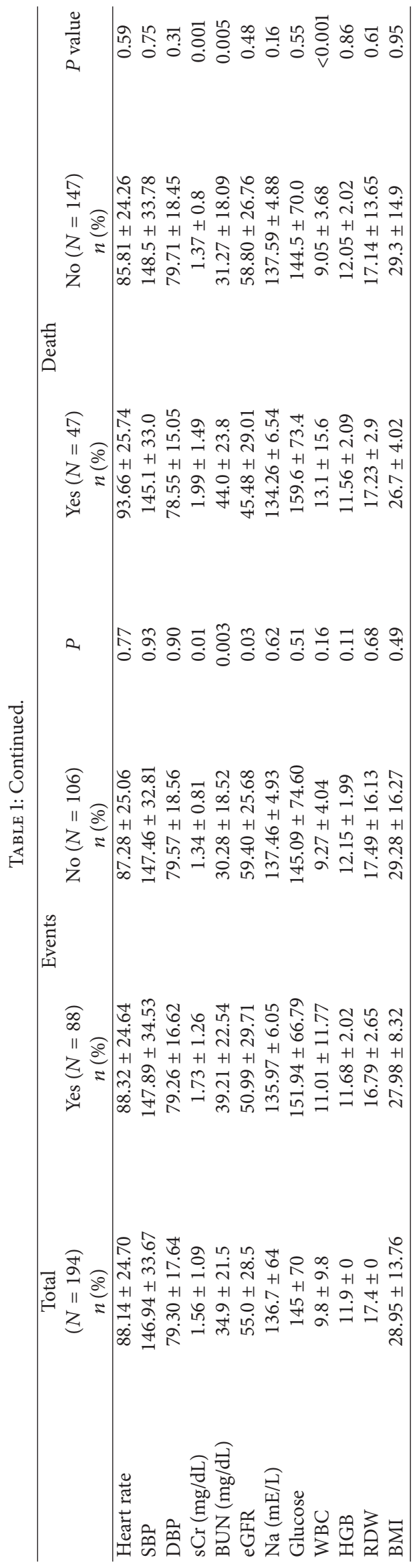




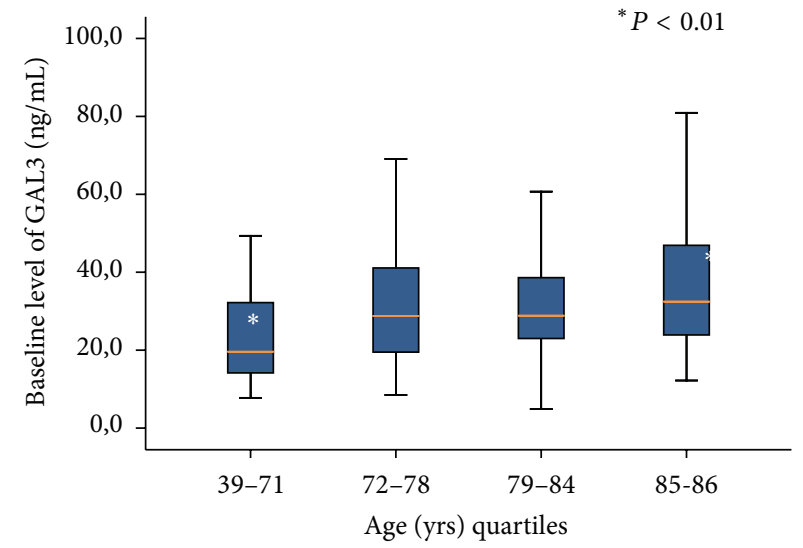

(a)

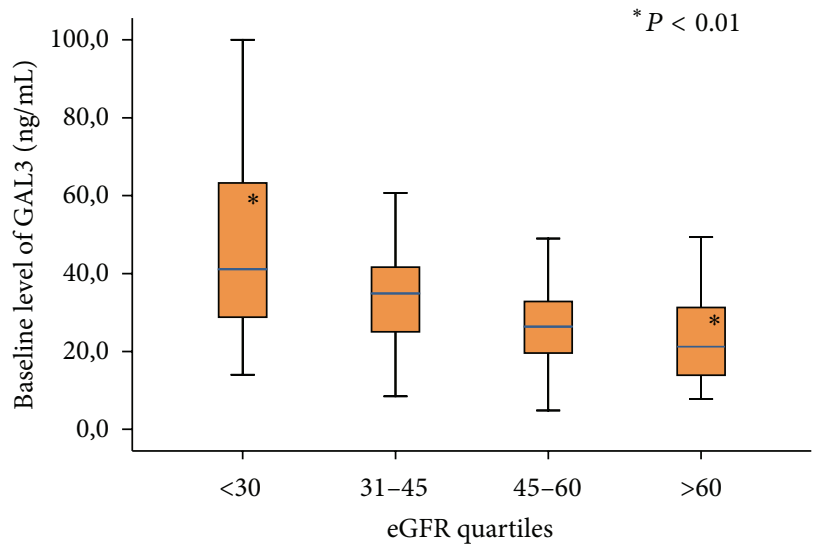

(b)

FIGURE 2: GAL3 levels increase proportionally with age quartiles (a) and decrease with eGFR quartiles (b).

TABLE 2: Baseline biomarkers as a function of the primary endpoint of events (in-hospital death and rehospitalization and death at 18-month follow-up) and death.

\begin{tabular}{lccccccc}
\hline \multirow{2}{*}{ Variable $($ mean $\pm \mathrm{SD})$} & \multicolumn{2}{c}{ Total } & \multicolumn{2}{c}{ Events (death + rehospitalization) } & \multirow{2}{*}{$P$} & \multicolumn{3}{c}{ Death } \\
& $(N=194)$ & Yes $(N=88)$ & No $(N=106)$ & & Yes $(N=47)$ & No $(N=147)$ \\
\hline GAL3 $(\mathrm{ng} / \mathrm{mL})$ & $32.19 \pm 19.03$ & $37.15 \pm 21.8$ & $26.12 \pm 13.6$ & $<0.001$ & $40.58 \pm 23.09$ & $28.16 \pm 15.96$ & $<0.001$ \\
BNP $(\mathrm{pg} / \mathrm{mL})$ & $872.9 \pm 1024.4$ & $969.5 \pm 1205.5$ & $774.5 \pm 837.1$ & 0.91 & $1030.0 \pm 1059.9$ & $821.25 \pm 1014.6$ & 0.27 \\
Hydration $(\%)$ & $79.44 \pm 6.55$ & $80.75 \pm 6.55$ & $78.75 \pm 6.66$ & 0.75 & $80.62 \pm 7.6$ & $79.05 \pm 6.19$ & 0.11 \\
Xc/H & $34.8 \pm 16.4$ & $27.0 \pm 14.2$ & $27.35 \pm 11.63$ & 0.93 & $28.25 \pm 26.85$ & $17.07 \pm 11.28$ & 0.05 \\
Rz/H & $364.25 \pm 136.8$ & $363.73 \pm 140.02$ & $341.07 \pm 120.88$ & 0.21 & $386.05 \pm 340.54$ & $155.30 \pm 119.68$ & 0.28 \\
Phase angle & $4.4 \pm 1.7$ & $4.3 \pm 1.7$ & $4.7 \pm 1.5$ & 0.17 & $4.24 \pm 2.09$ & $4.62 \pm 1.48$ & 0.79 \\
\hline
\end{tabular}

BIVA (phase angle), and GAL3 + phase angle for mortality and rehospitalization for 30,60, 90, and 180 days and 12 and 18 months. Figure 3 shows the Kaplan-Meier survival curve for death (a) and for total events (death and rehospitalization) (b) on the basis of GAL3 values greater than $17.8 \mathrm{ng} / \mathrm{mL}$, international cut-off [12]. It is evident that in patients with GAL3 $>17.8 \mathrm{ng} / \mathrm{mL}$ there was a higher incidence of death or of all events (death and rehospitalization) during 18month follow-up. Cox regression analysis demonstrated that GAL3 is an indipendent variable to predict death to predict death and rehospitalization with a value of $32.24 \mathrm{ng} / \mathrm{mL}$ at 30 days $(P<0.005)$. We constructed a clinical model based on variables suggested by the referee (age, sex, BNP, LVEF, and creatinine), using as decisional cut-off of the median values of our entire population. Each patient was classified at low or high risk for development of events on the basis of the positivity at the different predictive variables. The best predictive model in terms of sensitivity and specificity was obtained with positivity at 3 out of 5 variables (sensitivity $61.4 \%$, specificity $61.8 \%$, and accuracy $62 \%$ ). The use of galectin-3 at a threshold of 17.8 combined to previously described clinical model was able to improve the global risk classification (NRI) of $18 \%$ (NRI for events $+27 \%$, NRI for no events $-9 \%, P=0.021)$. The use of galectin-3 and BIVA was able to get a NRI of $20 \%$ especially improving the "no events" correct reclassification (NRI for events $+16 \%$, NRI for no events $+4 \%, P=0.012$ ).

\section{Discussion}

Cardiac remodeling and congestion are crucial determinants of the clinical outcome of heart failure (HF) and are linked to disease progression and poor prognosis [18]. Recently it has been demonstrated that slowing or reversing the progression of remodeling could become a therapeutic goal of HF patients' management. Circulating plasma concentrations of BNP is currently the most commonly used biomarker in $\mathrm{AHF}$ and its level is generally increased in proportion to the severity of the myocardial stretch or overload [19]. However, the applicability of BNP is limited, since its levels substantially vary over the day, and is not related to the underlying cardiac disease process. Prognostic value of GAL3 when upregulated in hypertrophied hearts has been confirmed in a number of studies [2, 3, 20-25]. Data from our study strongly support the original research papers that showed how GAL3 could play an important role in the underlying structural heart disease processes. Elevated value of GAL3 is associated with heart failure progression due to increase of heart fibrosis leading to poor outcome in AHF patients. 
TABLE 3: ROC curve analysis of GAL3, BIVA (phase angle), and GAL3 + phase angle for mortality and rehospitalization for 30, 60, and 90 days, 1 year, and 18 months.

\begin{tabular}{|c|c|c|c|c|c|c|}
\hline & \multicolumn{2}{|c|}{ GAL3 + phase angle } & \multicolumn{2}{|c|}{ GAL3 } & \multicolumn{2}{|c|}{ Phase angle } \\
\hline & AUC & $P$ & AUC & $P$ & AUC & $P$ \\
\hline \multicolumn{7}{|c|}{ Rehospitalization } \\
\hline 30 days & 0.526 & ns & 0.51 & ns & 0.52 & ns \\
\hline 60 days & 0.625 & 0.003 & 0.61 & 0.04 & 0.54 & 0.04 \\
\hline 90 days & 0.583 & ns & 0.57 & ns & 0.57 & ns \\
\hline 180 days & 0.545 & 0.05 & 0.54 & ns & 0.52 & ns \\
\hline 12 months & 0.52 & ns & 0.54 & ns & 0.53 & ns \\
\hline 18 months & 0.620 & 0.04 & 0.59 & 0.04 & 0.52 & ns \\
\hline \multicolumn{7}{|c|}{ Death } \\
\hline 30 days & 0.764 & 0.0001 & 0.69 & 0.002 & 0.64 & 0.01 \\
\hline 60 days & 0.754 & 0.0001 & 0.68 & 0.0001 & 0.68 & 0.003 \\
\hline 90 days & 0.667 & 0.005 & 0.64 & 0.01 & 0.58 & 0.04 \\
\hline 180 days & 0.841 & 0.0001 & 0.67 & 0.006 & 0.79 & 0.0001 \\
\hline 12 months & 0.833 & 0.0001 & 0.72 & 0.002 & 0.79 & 0.0001 \\
\hline 18 months & 0.863 & 0.0001 & 0.73 & 0.0003 & 0.86 & 0.0001 \\
\hline
\end{tabular}

TABLE 4: Multivariate Cox regression analysis GAL3 is an independent variable to predict death and rehospitalization with a value of $32.24 \mathrm{ng} / \mathrm{mL}$ at 30 days $(P<0.005)$.

\begin{tabular}{|c|c|c|c|c|}
\hline \multirow{2}{*}{ Variables } & \multicolumn{4}{|c|}{ Logistic regression } \\
\hline & $B$ & SE & Wald & $P$ \\
\hline GAL3 & 0.671 & 0.370 & 3.290 & 0.05 \\
\hline Phase angle & -1.462 & 0.773 & 3.574 & $<0.03$ \\
\hline $\mathrm{Hi}$ & 0.103 & 0.708 & 0.021 & ns \\
\hline
\end{tabular}

From our results GAL3 value in patients who develop higher incidence of death and rehospitalization was independent risk factor with a cut-off of $32.24 \mathrm{ng} / \mathrm{mL}$ greater than the cutoff of $17.8 \mathrm{ng} / \mathrm{mL}$ that is currently accepted, Table $4[12,20-$ 26]. Moreover GAL3 was statistically higher in patients who died $(P<0.001)$ and were rehospitalized compared to survivors $(P<0.001)$. Our results are also in agreement with van Kimmenade et al. who demonstrated that, elevated GAL3 levels in patients presenting to ED for AHF, GAL3 was the best independent predictor for 60-day mortality [4]. Furthermore our results are in the same directions with the ones from Shah et al. who demonstrated that in patients with AHF presenting to ED a value of GAL3 levels above the median value had higher incidence of mortality [3]. Our study is probably the first in which a long-term follow-up for mortality and rehospitalization has been studied in AHF patients in consideration of GAL3 levels measured in ED; furthermore, our follow-up study was serially conducted (30, 60-, 90-, and 180-day and 12- and 18-month follow-up events (deaths or rehospitalization)). In literature there are very few data on short- and long-term follow-up but all these papers are not serially $[3,4,27]$. Recently published data from three large research trials from HF patients. They demonstrated that elevation of GAL3 levels was significantly predictive of rehospitalization of 30 days from discharge. Moreover those patients of elevation of GAL3 at the time of hospitalization were readmitted within 30 days at three times of rate of patients without GAL3 elevation and the increased risk of hospitalization conferred by GAL3 elevation persisted at 60, 90, and 120 days in the study [28].

As described in literature GAL3 was able to identify those AHF patients at risk for short-term death or for the combination of death and readmission [4]; in our study GAL3 measured at the moment of ED arrivals predicts adverse events better than BNP. So we can consider that on the basis of this data BNP has not a real prognostic value at admission; our group already demonstrated this finding in the ITALIAN RED study [29]. However, we must underline the fact that BNP is internationally considered as the gold standard biomarker in any risk score of AHF patients.

We also analyzed the levels of GAL3 based on eGFR quartiles and we demonstrated that GAL3 levels were significantly higher in patients with reduced eGFR $\left(<30 \mathrm{~mL} / \mathrm{min} / 1.73 \mathrm{~m}^{2}\right)$. O'Seaghdha et al. already demonstrated that high levels of GAL3 were associated with a rapid decline of eGFR and with a higher risk of incidence of chronic kidney disease (CKD) [30]. Findings from our study are also supported by a study from Tang et al. who recently demonstrated that high GAL3 levels are associated with a poor renal function [24]. As a consequence, the inverse relationship between GAL3 and renal function, which we observed in our AHF patients, leads to the suggestion that increased plasma GAL3 in AHF might be linked to renal dysfunction, and the ability of GAL3 to predict outcomes in HF might reflect the consequences of renal impairment $[3,24,25]$. Moreover, GAL3 levels were significantly higher in patients with age $>71$ years, too. This 


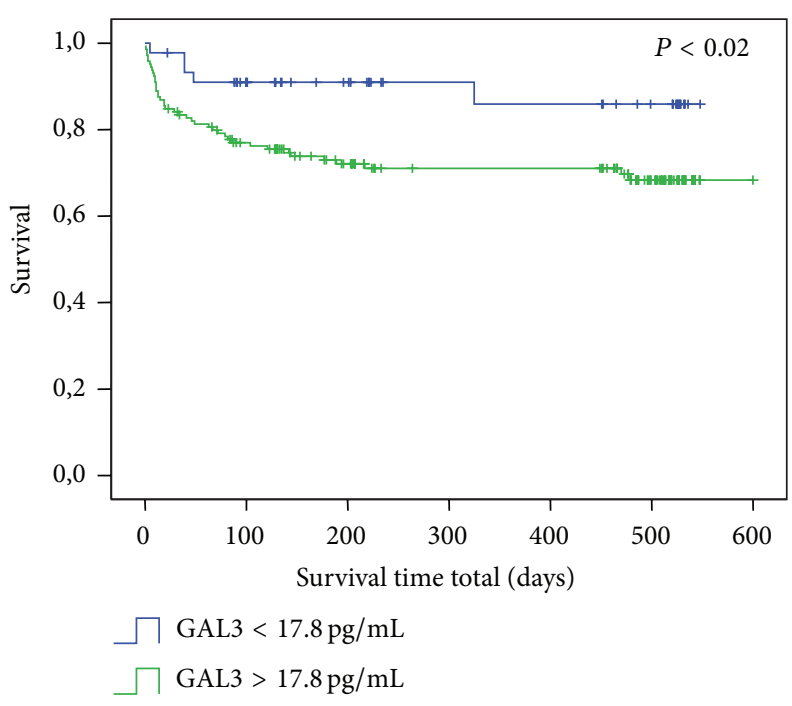

(a)

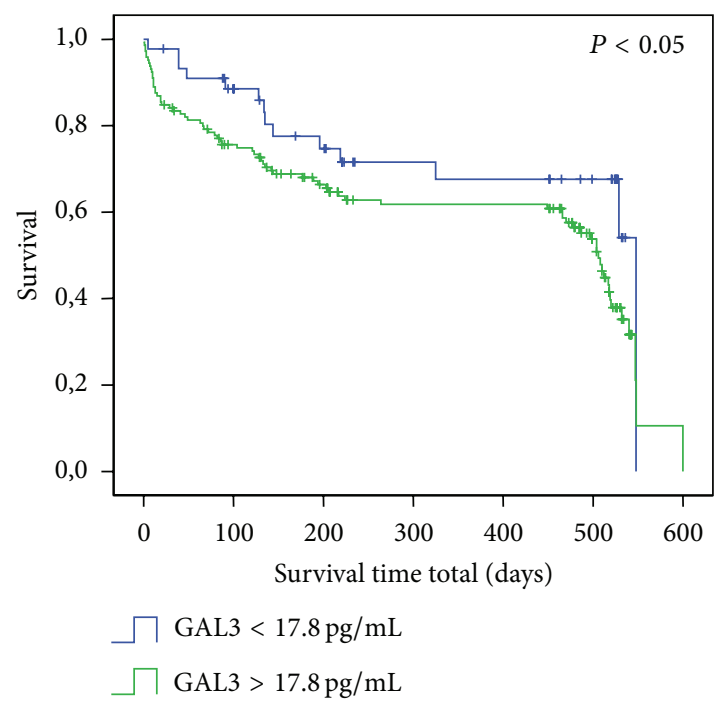

(b) Kaplan-Meier GAL3 for all events (death + rehospitalization)

Figure 3: Kaplan-Meier for death (a) and events (b) on the basis of GAL3 values greater than $17.8 \mathrm{ng} / \mathrm{mL}$.

suggests that in evaluating the value of GAL3 the physician should take into account the age of patients as confounding factor. This association of elevated GAL3 level and age should not be surprising; it is well known that heart fibrosis is a structural process related to aging. Other authors already found this relationship with GAL3 and age in the same cohort of patients [24]. We also found that BIVA had significant prognostic value for death for each considered follow-up time. This is not surprising and confirms previous study of our group, we also demonstrated additive value for AHF risk stratification of BIVA + BNP $[8,9]$. The most important of our opinion results of our study is that the combination of BIVA + GAL3 increases the prognostic value for death and also for rehospitalization. It seems that the prognostic value of the combining of BIVA and GAL3 is of great significance since they mirror two different physiopathological aspects of HF. BIVA (phase angle) represents the degree of body fluid congestion, typical of AHF patients especially in the end stage of the disease; on the other hand, GAL3 value represents the level of fibrosis and the heart remodeling [31]. The combination of BIVA (phase angle) and GAL3 seems to have the better AUC compared to BIVA alone or GAL3 alone in predicting patients' adverse outcome. Nevertheless in our study multivariate Cox regression analysis showed that GAL3 is an independent variable more than BIVA variables to predict death and rehospitalization of value $32.24 \mathrm{ng} / \mathrm{mL}$ only at 30 days. These results strengthen the hypothesis that GAL3 could be considered a better predictive marker in comparison to BIVA and BNP when measured at admission in ED. More studies on larger population group are needed to confirm this hypothesis. When the survival Kaplan-Meier curve was made for GAL3, we found that, considering the cut-off of $17.8 \mathrm{ng} / \mathrm{mL}$, those patients above this cut-off had a significant higher incidence of death and rehospitalization. This confirms the importance of cut-off of
GAL3 of $17.8 \mathrm{ng} / \mathrm{mL}$ representing an additive value to help physicians especially emergency physicians to individuate those patients of adverse outcomes.

\section{Conclusion}

In patients admitted for AHF an early assessment of GAL3 and BIVA seems to be useful in identifying patients at high risk for death and rehospitalization at short and long term. Combining the biomarker and the device could be of great utility since they are monitoring the severity of two pathophysiological different mechanisms underlining the severity of disease: heart fibrosis and fluid overload. GAL3 per se has the strongest independent predicting value for death and rehospitalization at 30 days with a cut-off of $32.24 \mathrm{ng} / \mathrm{mL}$. The use of combining GAL3 and BIVA assessment, at the moment of AHF patients' presentation, could support decision making and different therapeutic approach.

\section{Conflict of Interests}

The authors declare that there is no conflict of interests regarding the publication of this paper.

\section{Acknowledgment}

The authors want to acknowledge all authors for their helpful contribution to the development of the scientific work on behalf of GREAT international.

\section{References}

[1] H. M. Krumholz, A. R. Merrill, E. M. Schone et al., "Patterns of hospital performance in acute myocardial infarction and 
heart failure 30-day mortality and readmission," Circulation: Cardiovascular Quality and Outcomes, vol. 2, no. 5, pp. 407-413, 2009.

[2] A. S. Desai and L. W. Stevenson, "Rehospitalization for heart failure: predict or prevent?" Circulation, vol. 126, no. 4, pp. 501506, 2012.

[3] R. V. Shah, A. A. Chen-Tournoux, M. H. Picard, R. R. van Kimmenade, and J. L. Januzzi, "Galectin-3, cardiac structure and function, and long-term mortality in patients with acutely decompensated heart failure," European Journal of Heart Failure, vol. 12, no. 8, pp. 826-832, 2010.

[4] R. R. van Kimmenade, J. L. Januzzi Jr., P. T. Ellinor et al., "Utility of amino-terminal pro-brain natriuretic peptide, galectin-3, and apelin for the evaluation of patients with acute heart failure," Journal of the American College of Cardiology, vol. 48, no. 6, pp. 1217-1224, 2006.

[5] E. López, V. del Pozo, T. Miguel et al., "Inhibition of chronic airway inflammation and remodeling by galectin-3 gene therapy in a murine model," The Journal of Immunology, vol. 176, no. 3, pp. 1943-1950, 2006.

[6] M. Gheorghiade, F. Follath, P. Ponikowski et al., "Assessing and grading congestion in acute heart failure: a scientific statement from the acute heart failure committee of the heart failure association of the European society of cardiology and endorsed by the European society of intensive care medicine," European Journal of Heart Failure, vol. 12, no. 5, pp. 423-433, 2010.

[7] A. Piccoli, M. Codognotto, V. Cianci et al., "Differentiation of cardiac and noncardiac dyspnea using bioelectrical impedance vector analysis (BIVA)," Journal of Cardiac Failure, vol. 18, no. 3, pp. 226-232, 2012.

[8] S. Di Somma, B. de Berardinis, C. Bongiovanni, R. Marino, E. Ferri, and B. Alfei, "Use of BNP and bioimpedance to drive therapy in heart failure patients," Congestive Heart Failure, vol. 16, no. supplement 1, pp. S56-S61, 2010.

[9] S. Di Somma, I. Lalle, L. Magrini et al., "Additive diagnostic and prognostic value of Bioelectrical Impedance Vector Analysis (BIVA) to brain natriuretic peptide "grey-zone" in patients with acute heart failure in the emergency department," European Heart Journal: Acute Cardiovascular Care, vol. 3, no. 2, pp. 167175, 2014.

[10] J. J. McMurray, S. Adamopoulos, S. D. Anker et al., "ESC Guidelines for the diagnosis and treatment of acute and chronic heart failure 2012: the task force for the diagnosis and treatment of acute and chronic heart failure 2012 of the European Society of Cardiology. Developed in collaboration with the Heart Failure Association (HFA) of the ESC," European Heart Journal, vol. 33, no. 14, pp. 1787-1847, 2012.

[11] A. S. Levey, J. P. Bosch, J. B. Lewis, T. Greene, N. Rogers, and D. Roth, "A more accurate method to estimate glomerular filtration rate from serum creatinine: a new prediction equation. Modification of diet in Renal Disease Study Group," Annals of Internal Medicine, vol. 130, no. 6, pp. 461-470, 1999.

[12] P. A. McCullough, A. Olobatoke, and T. E. Vanhecke, "Galectin3: a novel blood test for the evaluation and management of patients with heart failure," Reviews in Cardiovascular Medicine, vol. 12, no. 4, pp. 200-210, 2011.

[13] D. J. Whellan, C. M. O'Connor, K. L. Lee et al., "Heart failure and a controlled trial investigating outcomes of exercise training (HF-ACTION): design and rationale," American Heart Journal, vol. 153, no. 2, pp. 201-211, 2007.

[14] R. Valle, N. Aspromonte, L. Milani et al., "Optimizing fluid management in patients with acute decompensated heart failure
(ADHF): the emerging role of combined measurement of body hydration status and brain natriuretic peptide (BNP) levels," Heart Failure Reviews, vol. 16, no. 6, pp. 519-529, 2011.

[15] R. F. Kushner, "Bioelectrical impedance analysis: a review of principles and applications," Journal of the American College of Nutrition, vol. 11, no. 2, pp. 199-209, 1992.

[16] A. Piccoli, "Bioelectric impedance vector distribution in peritoneal dialysis patients with different hydration status," Kidney International, vol. 65, no. 3, pp. 1050-1063, 2004.

[17] H. C. Lukaski, P. E. Johnson, W. W. Bolonchuk, and G. I. Lykken, "Assessment of fat-free mass using bioelectrical impedance measurements of the human body," The American Journal of Clinical Nutrition, vol. 41, no. 4, pp. 810-817, 1985.

[18] R. A. de Boer, L. Yu, and D. J. van Veldhuisen, "Galectin-3 in cardiac remodeling and heart failure," Current Heart Failure Reports, vol. 7, no. 1, pp. 1-8, 2010.

[19] A. Maisel, C. Mueller, K. Adams Jr. et al., "State of the art: using natriuretic peptide levels in clinical practice," European Journal of Heart Failure, vol. 10, no. 9, pp. 824-839, 2008.

[20] R. A. de Boer, A. A. Voors, P. Muntendam, W. H. van Gilst, and D. J. van Veldhuisen, "Galectin-3: a novel mediator of heart failure development and progression," European Journal of Heart Failure, vol. 11, no. 9, pp. 811-817, 2009.

[21] H. Milting, P. Ellinghaus, M. Seewald et al., "Plasma biomarkers of myocardial fibrosis and remodeling in terminal heart failure patients supported by mechanical circulatory support devices," Journal of Heart and Lung Transplantation, vol. 27, no. 6, pp. 589-596, 2008.

[22] R. A. de Boer, D. J. Lok, T. Jaarsma et al., "Predictive value of plasma galectin-3 levels in heart failure with reduced and preserved ejection fraction," Annals of Medicine, vol. 43, no. 1, pp. 60-68, 2011.

[23] M. Lainscak, A. P. Coletta, N. Sherwi, and J. G. Cleland, “Clinical trials update from the Heart Failure Society of America Meeting 2009: FAST, IMPROVE-HF, COACH galectin-3 substudy, HFACTION nuclear substudy, DAD-HF, and MARVEL-1," European Journal of Heart Failure, vol. 12, no. 2, pp. 193-196, 2010.

[24] W. H. Tang, K. Shrestha, Z. Shao et al., "Usefulness of plasma galectin-3 levels in systolic heart failure to predict renal insufficiency and survival," American Journal of Cardiology, vol. 108, no. 3, pp. 385-390, 2011.

[25] D. J. A. Lok, P. van der Meer, P. W. de la Porte et al., "Prognostic value of galectin-3, a novel marker of fibrosis,in patients with chronic heart failure: data from the DEAL-HF study," Clinical Research in Cardiology, vol. 99, no. 5, pp. 323-328, 2010.

[26] A. R. van der Velde, L. Gullestad, T. Ueland et al., "Prognostic value of changes in galectin-3 levels over time in patients with heart failure data from CORONA and COACH," Circulation: Heart Failure, vol. 6, no. 2, pp. 219-226, 2013.

[27] A. Bayes-Genis, M. de Antonio, J. Vila et al., "Head-to-head comparison of 2 myocardial fibrosis biomarkers for long-term heart failure risk stratification: ST2 versus galectin-3," Journal of the American College of Cardiology, vol. 63, no. 2, pp. 158-166, 2014.

[28] W. C. Meijers, J. L. Januzzi, C. deFilippi et al., "Elevated plasma galectin-3 is associated with near-term rehospitalization in heart failure: a pooled analysis of 3 clinical trials," American Heart Journal, vol. 167, no. 6, pp. 853.e4-860.e4, 2014.

[29] S. Di Somma, L. Magrini, V. Pittoni et al., "In-hospital percentage BNP reduction is highly predictive for adverse events in patients admitted for acute heart failure: the Italian RED Study," Critical Care, vol. 14, no. 3, article R116, 2010. 
[30] C. M. O'Seaghdha, S. J. Hwang, J. E. Ho, R. S. Vasan, D. Levy, and C. S. Fox, "Elevated galectin-3 precedes the development of CKD," Journal of the American Society of Nephrology, vol. 24, no. 9, pp. 1470-1477, 2013.

[31] U. C. Sharma, S. Pokharel, T. J. van Brakel et al., "Galectin-3 marks activated macrophages in failure-prone hypertrophied hearts and contributes to cardiac dysfunction," Circulation, vol. 110, no. 19, pp. 3121-3128, 2004. 

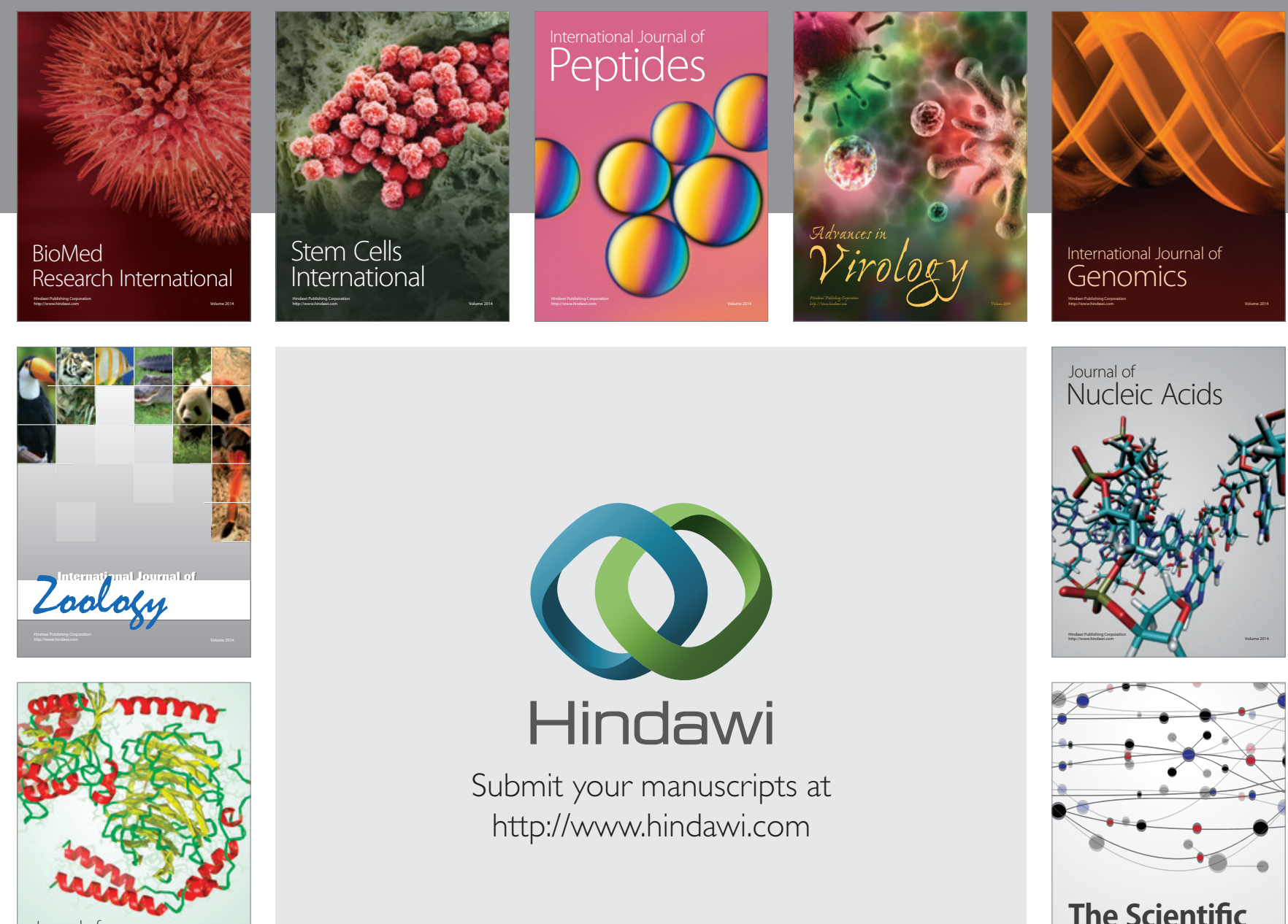

Submit your manuscripts at

http://www.hindawi.com

Journal of
Signal Transduction
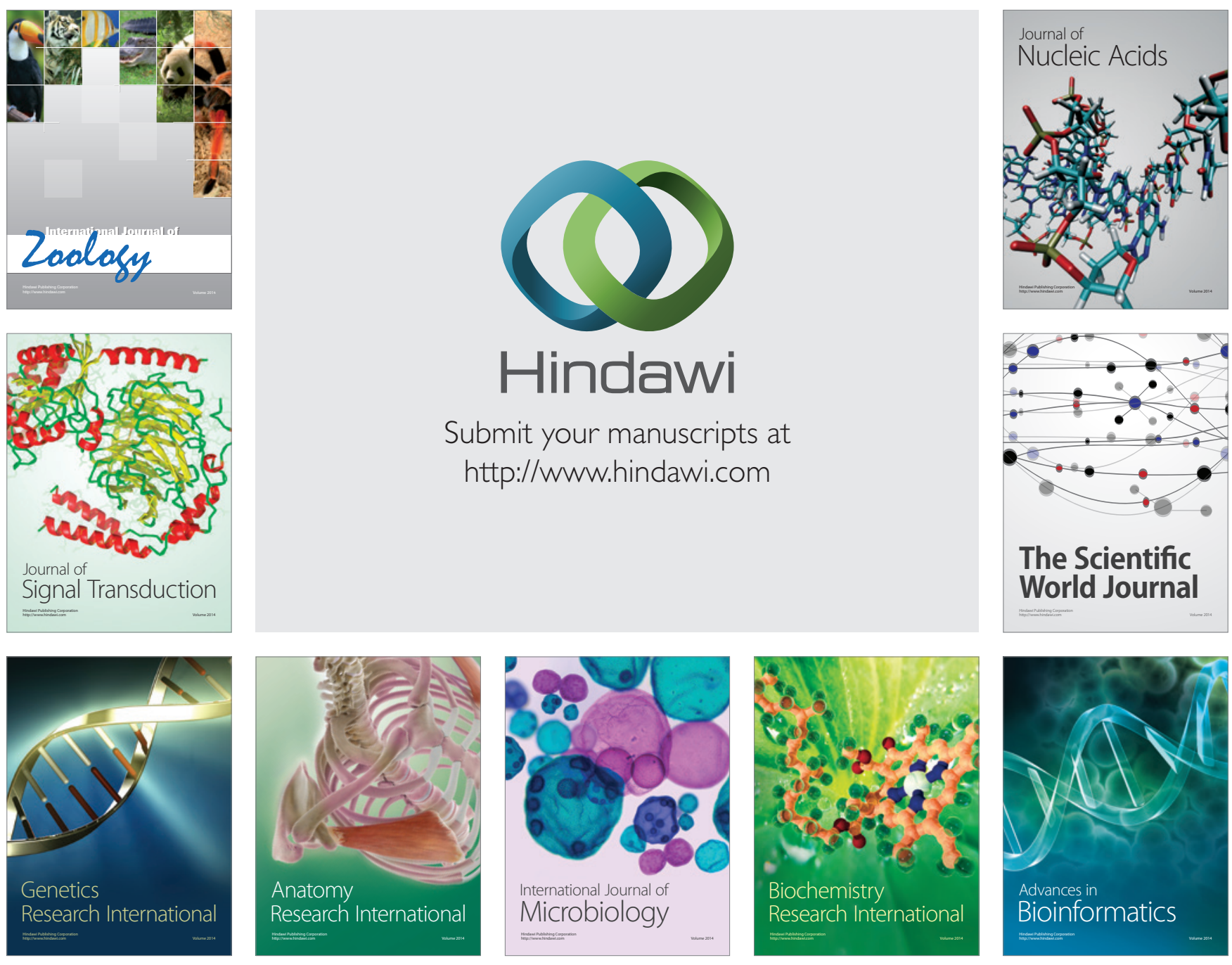

The Scientific World Journal
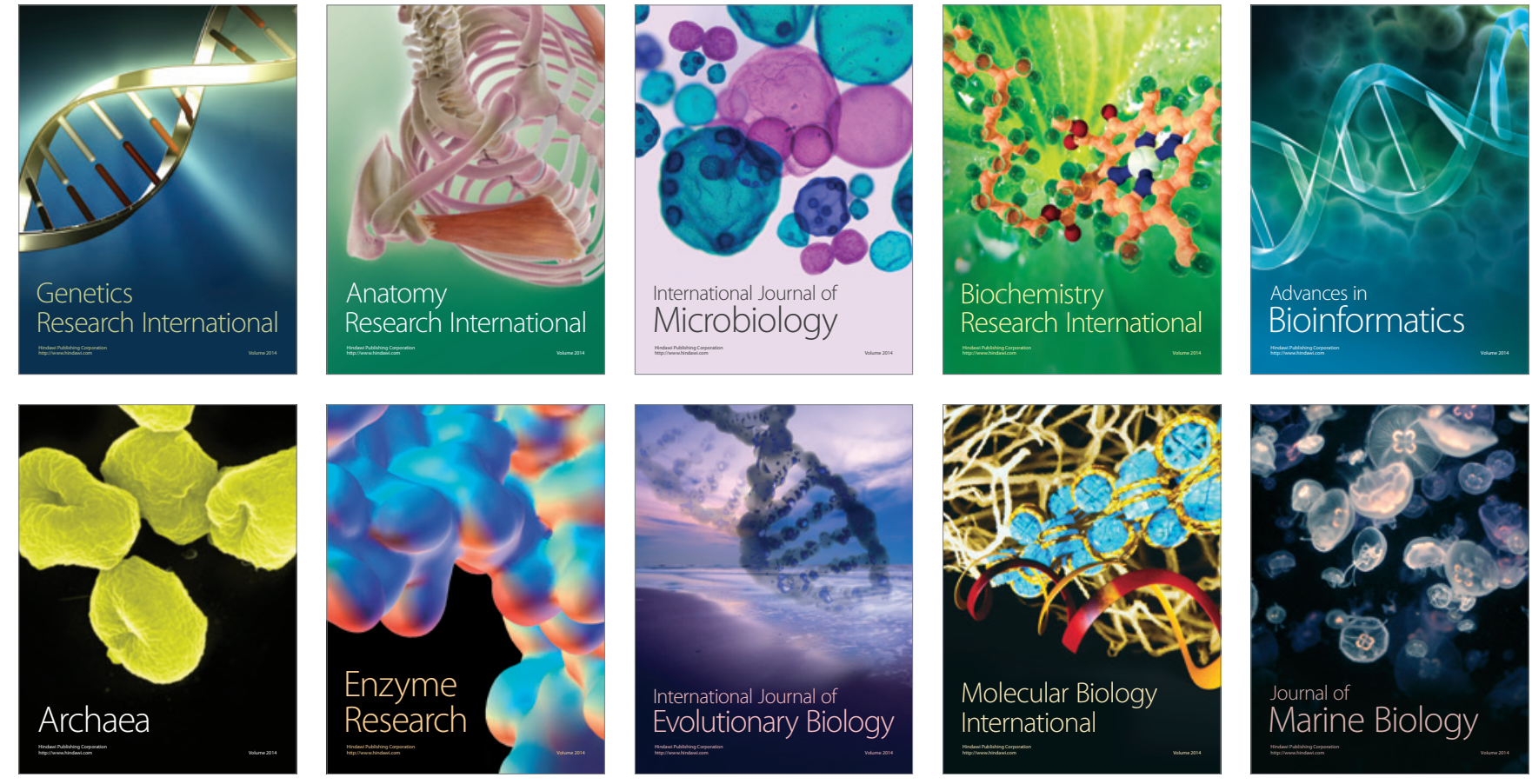